\title{
MONITORING THE DETERIORATION OF STONE AT MINDENER MUSEUM'S LAPIDARIUM
}

\author{
Guenter Pomaska \\ Faculty for Architecture and Civil Engineering, University of Applied Sciences Bielefeld, Germany - \\ gp@imagefact.de
}

XXIV International CIPA Symposium SR3

KEY WORDS: Acquisition, Modelling, Close Range, Internet/WEB, Reconstruction, Archaeology

\begin{abstract}
:
Mindener Museum's Lapidarium incorporates a collection of stone work like reliefs, sculptures and inscriptions from different time epochs as advices of the city's history. These gems must be protected against environmental influences and deterioration. In advance of the measures a 3D reconstruction and detailed documentation has to be taken. The framework to establish hard- and software must match the museum's infrastructure. Two major question will be answered. Are low-cost scanning devices like depth cameras and digital of the shelf cameras suitable for the data acquisition? Does the functionality of open source and freeware covers the demand on investigation and analysis in this application?
\end{abstract}

The working chain described in this contribution covers the structure from motion method and the reconstruction with RGB-D cameras. Mesh processing such as cleaning, smoothing, poisson surface reconstruction and texturing will be accomplished with MeshLab. Data acquisition and modelling continues in structure analysis. Therefore the focus lies as well on latest software developments related to 3D printing technologies. Repairing and finishing of meshes is a task for MeshMixer. Netfabb as a tool for positioning, dimensioning and slicing enables virtual handling of the items. On the Sketchfab web site one can publish and share 3D objects with integration into web pages supported by WebGL. Finally if a prototype is needed, the mesh can be uploaded to a 3D printing device provided by an online service.

\section{RECORDING OF UNSTRUCTURED OBJECTS}

Unstructured objects like reliefs, sculptures or figurines are being recorded today as high density point clouds or surface meshes. The geometric representation is a polygon surface that consists of small triangles or quads while the radiometric information is given by grey values for the vertices or texture coordinates referenced to bitmaps. Scanning devices are available as laser scanning, structured light methods, computer vision methods with RGB sensors, time of flight cameras or depth cameras. Criteria for selecting the appropriate technology and method depends on the shape of the object, the measurement volume, accessibility, light and surface conditions, required accuracy and as well in terms of operating efficiency and budget.

Laser scanning, known as pulse measurement or phase comparison, covers a measurement volume from 1 to 1000 metre within an accuracy range from millimetre to centimetre. Combinations of laser light and RGB cameras are known as structured light scanning. With the latter one can cover distances from nearly 0 to some meters, achieving very high accuracy within micro millimetre range. Time of flight cameras provide measurement volumes up to 10 metre with high frequency of $100 \mathrm{fps}$ but are not in competition with structured light methods due to the low resolution and poor accuracy. Latest developments of depth cameras from Primesense, an Israeli company, designed for human natural interaction with digital devices enable close range 3D surface recording in real time using low-cost equipment. The frame rate of a depth camera may be around 30fps, measurement distance is from 0,4 up to some meters, the accuracy in depth can be announced in the range of a centimetre.

The most flexible method on site is provided by photogrammetric and computer vision methods known as structure from motion or structure from stereo. Range and precision of a mesh depends on camera parameter and exposure arrangement.

In the here presented study on documentation of stone works, web cams and digital cameras provide images for the structure from motion method. The Kinect RGB-D camera from Microsoft and the Primensense Carmine 1.09 driven by standard software tools are tested on a practical approach. Figure 1 displays a fraction of the stone collection (lapidarium) recorded with the structure from motion method. The scene is reconstructed by 123D Catch with 142100 triangles.

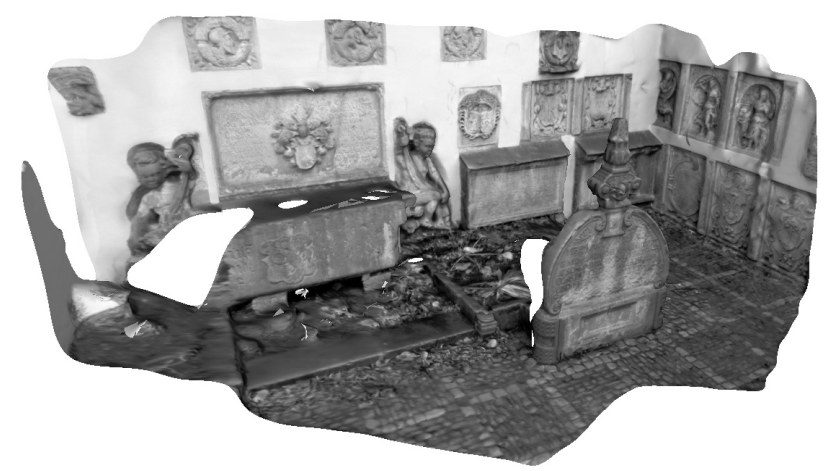

Figure 1: Part of Mindener Museum's lapidarium - textured mesh from Autodesk 123DCatch 


\section{RGB-DEPTH CAMERAS}

\subsection{Hardware}

Primesense Carmine, Asus Xtion and Kinect for Windows are more or less based on the same construction principle. An infrared projector, an infra-red camera and a RGB sensor are the eyes of the equipment, while the Primesense light coding technology on one chip is the heart. We don't consider the Audio components in this paper. Figure 2 (source www.primsense.com) displays a diagram of the basic components.

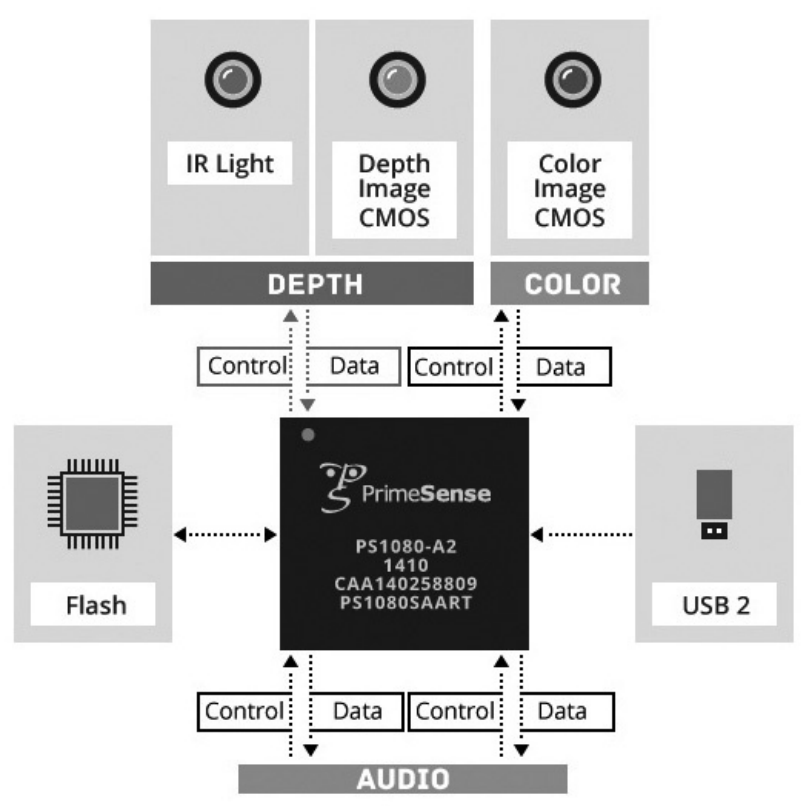

Figure 2: Elektronic components of a Carmine sensor

The infra-red projector configures a point pattern to the object. Comparison between the outgoing and the incoming pattern delivers a depth image of $640 \times 480$ px with 11 bit memory depth. RGB color information has to be transformed onto the depth image based on the calibration of the components. A photo taken with an infra-red camera visualizes the pattern on the object, see figure 3. The equipment on site is a notebook and a Kinect for Windows camera. The table plate mounted on the tripod carries the notebook and enables a smooth movement of the camera.

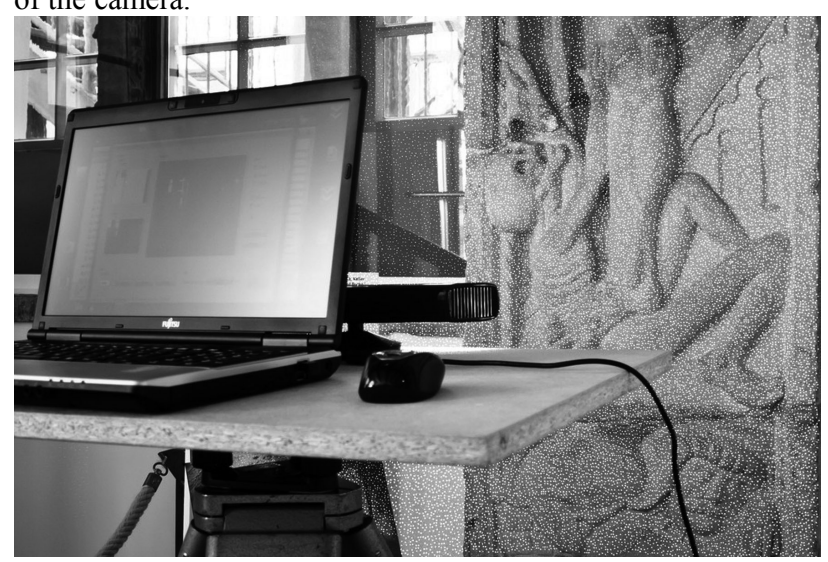

Figure 3: Infrared photo showing the point pattern and the Kinect on site in operation
It has to be mentioned that the Kinect needs its own power supply while Asus and Primesense both take the power from the USB port. Measurement volume of the cameras is a cube from $0.4 \mathrm{~m}$ up to $10 \mathrm{~m}$ with decreasing depth accuracy. Primesense announces for their Carmine 1.08 a depth resolution of around $12 \mathrm{~mm}$ at $2 \mathrm{~m}$ distance and for the version 1.09 in the near distances of about $0.5 \mathrm{~m}$ a resolution of $1 \mathrm{~mm}$. Due to the USB power connection, the much smaller size and weight and the higher accuracy we decided to apply for the present application the Primesense Carmine 1.09.

A steady cam like camera stabilizer carries the sensor for a smoother free hand movement. Using VNC (virtual network computing) technology facilitates viewing and software control via a smartphone's touch screen. The VNC server UltraVNC is running on the notebook and the AndroidVNC viewer on the smartphone. It turns the smartphone into a control unit. Connection of both components is given by the mobile hotspot and wireless LAN connected to Android hotspot. Field operation in this way is independent from other network resources. A prototype of this kind of equipment is demonstrated in figure 4.

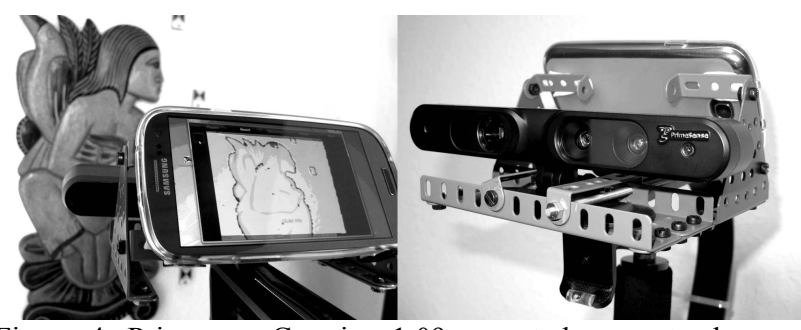

Figure 4: Primesene Carmine 1.09 mounted on a steady cam together with a smartphone as a control unit

\subsection{Recording Software for RGB-D Cameras}

Scanning software for depth cameras is available in different flavours for Windows systems. Supported are the OpenNI device driver and the Microsoft SDK Kit and driver. Usually free versions of the application software are coming with some limitations while the paid versions request a moderate license fee. The scanning principles are all similar. Meshes between frames from moving the camera or rotating the object are aligned in real time. Finishing the mesh differs from system to system. Hardware recommendations are in most cases 64Bit, Windows 7 or higher and Nvidia CUDA compatible graphic boards. Some software is running under Windows XP on a Nvidia GTX 240 graphics card. But due to the limited performance this configuration is not recommended. It is not the place here to cover all available software products. At the time of writing this contribution amongst others MS Kinect Fusion, ReconstructMe, Brekel Kinect, Artec 3D Studio, KScan3D, Skanect, Scenect from Faro they all did their job. We tested some software packages for their suitability. To the early released systems belonged ReconstructMe and a kind of beta version of Skanect. In addition KScan3D created some interest.

RecontructMe (in short ReMe) is a 3D reconstruction system from an Austrian company. It is available as a console software with a graphical user interface or SDK for Windows platforms. Free non-commercial license is given for the console version and personal use. The settings for hardware, resolution and measurement volume must be predefined in a file. The software initializes the sensor and starts localising and tracking objects in the predefined volume. Two keys are needed for recording, $\mathrm{P}$ for pause and $\mathrm{R}$ for resetting the volume, that's all. ESC ends 
the data acquisition. Output formats are OBJ, PLY, STL and 3DS. It is not only advertising if one states that ReconstructMe is powerful and easy to use.

\subsubsection{Skanect}

Skanect is a software from Manctl company, a French-American entity founded in late 2011. In June 2013 Skanect was acquired by Occipital, Inc. a developer of computer-vision based mobile apps. The software, here applied as version 1.4.0, supports Primesense, Asus Xtion and Kinect depth cameras for capturing a full colour 3D model of an object and creates 3D meshes. Skanect offers five menu tabs: Prepare, Record, Reconstruct, Process, Share. Preparing a scan requires some settings for the volume of the object, a path for file saving or reading settings from predefined files.

During the scan process the camera may be in motion or the object is positioned on a turntable. Matching the meshes is provided in real time, the covered object area for a scan is marked in green colour on the screen. Areas not reached by the scanning process are marked in red colour. Particular this kind of indication is very helpful during the scanning process. The operator can concentrate of moving the camera into the accurate position. Every frame of the scan, RGB as JPG and depth map as FSX format, is stored to offer offline reconstruction again after the scanning process. In case of small objects some clutters like bricks or boxes should be added to the scene to support the mesh matching. Some Bricks are shown in figure 5 on the turntable. PLY files can be edited with external software, MeshLab for instance, and reloaded into a project. Post processing procedures like hole filling or watertight generation, mesh simplification and colouring could finalize the mesh. Remarkable is the plane cutting function for closing a figurine. Direct export functions for sharing the mesh on Sketchfab and 3D printing with Sculpteo or exporting as PLY files can be called from the Share menu.

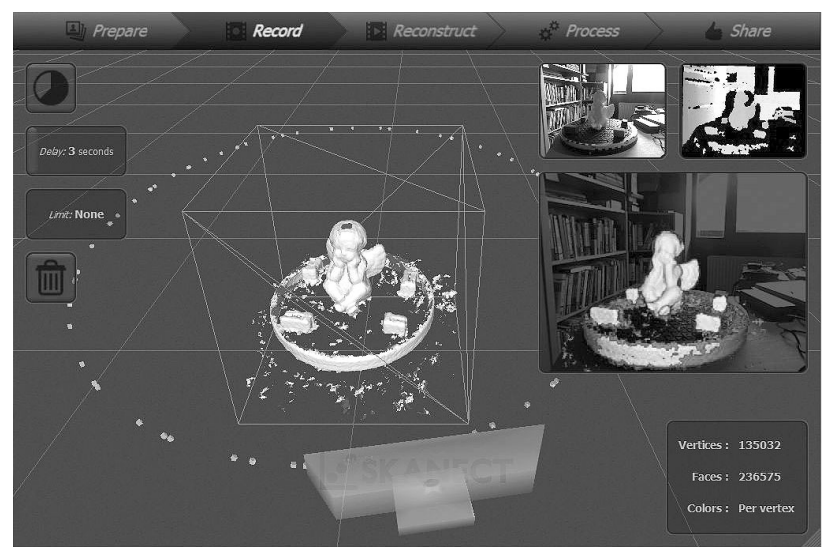

Figure 5: Skanect interface: 3D model, RGB and depth image

Skanect is available as a test version and a pro version for Windows 32Bit and 64Bit. CPU and GPU reconstruction is performed for CUDA 2.0 compatible graphic cards. OpenNI driver and Kinect for Windows SDK are both supported. With Skanect a fast and easy to handle software can be recommended.

\subsubsection{KScan3D}

KScan3D from LMI Technologies, Canada is a multi sensor system and differs in the scanning process and finalizing functions slightly from Skanect. The scanning mode can be used in manual and automatic alignment or in batch mode. Every scan is available separately. After aligning the single scans, groups can be combined and finalized with several settings for the amount of hole fillings, density and decimation of the number of faces. Using a lasso mode enables comfortable editing of the point cloud. KScan3D is simple and easy to handle as well. Figure 6 is a screen shot displaying the singles scans and the 3D view with the manual aligned single scans. The mesh is still in a kind of raw status marked with false colours as usual. On the left is the meshing and scanning panel. The measurement volume setting are realized by sliders. In advance of alignment unneeded areas are easy to select for elimination.

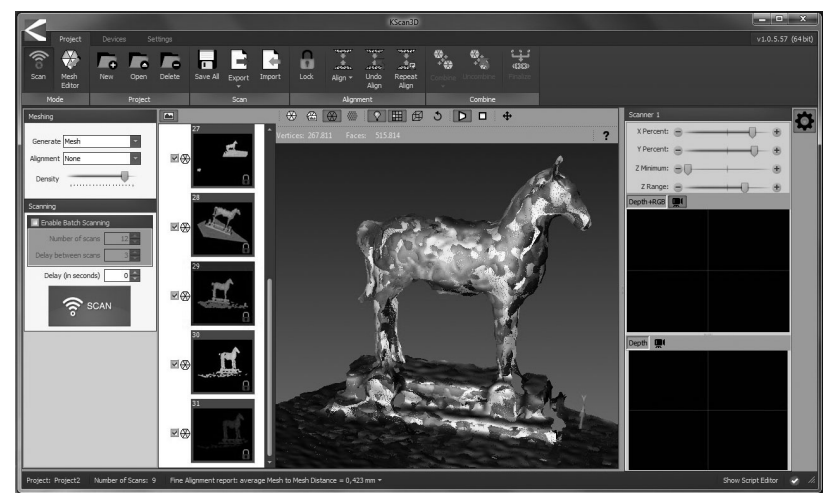

Figure 6: Recording 3D objects with KScan3D

Both software products Skanect and KScan3D are coming with some pros and cons but complete each other to a toolbox, fulfilling the requirements of our targeted application.

Much more other software suppliers provide useable tools for data acquisition with RGB-D cameras. Faro offers a free software Scenect for 64 bit Windows based on the professional laser scan software SCENE. Scanning with RGB-D cameras is integrated in the point cloud viewer Scene LT and supplied as freeware. The Faro software is branded as scanning for fun. Other free solutions are available with the Kinect Fusion project from Microsoft or Brekel Kinect, Jasper Brekelmans company located in Amsterdam. One can observe that the applications of $3 \mathrm{D}$ reconstruction applying Primesense technology is fast growing. Primensense already announced with Capri a new device coming soon. This indicates a rapid future development of depth cameras and related software.

\section{STRUCTURE FROM MOTION}

In computer vision structure from motion (SFM) indicates the process detecting spatial structures of objects due to corresponding features from images. SFM targets to the use of non calibrated cameras and totally automatically model reconstruction. The final object geometry is given as a sparse or dense colored point cloud including the camera positions and intrinsic parameter. Access to software is available as freeware distributions or online web services.

The working chain denotes with three corner stones: feature extraction, matching and adjustment. SIFT scale invariant feature is an algorithm for description and detection of features in images. Developed in 1999 by David Lowe at University of British Columbia. Lowe's method for image feature generation transforms an image into a large collection of feature vectors, each of which is invariant to image translation, scaling, and ro- 
tation, partially invariant to illumination changes and robust to local geometric distortion (source: http://en.wikipedia.org/wiki/Scaleinvariant_feature_transform. The second step is matching those features by finding the corresponding points in different images. The correspondence problem often is solved applying the iterative RANSAC method, first published by Fischler and Bolles in 1981 (see http://en.wikipedia.org/wiki/RANSAC). The key points and matches are taken by Bundler for calculating camera parameter, image positions and object points. The bundle adjustment program was supported by Microsoft Research and is available on the web under phototour.cs.washington.edu/Bundler. The Photosynth Web service has its roots in Bundler.

\subsection{Visual Structure from Motion VSFM}

Amongst several distributions as command line tools for 32Bit and 64Bit Windows computers, Visual SFM from Changchang $\mathrm{Wu}$, distinguishes as a powerful tool on Windows platforms.

VSFM integrates PVSM and CVSM and can improve the sparse point cloud to a dense point cloud. A comparison between both clouds can be observed in figure 7 . This sample is taken with a simple web cam without any approximate focal length values from the EXIF information. The background is not of interest, but covered with pages from a computer magazine for getting enough key points. The object itself only covers a very small part of an image. Surroundings stabilize the orientation procedure as well as the bricks mentioned in figure 5. In the left part of figure 7 the 21 camera positions are marked. Access to the calculation results is performed via the file bundler.out that is used as an interface to Bundler from other sources.
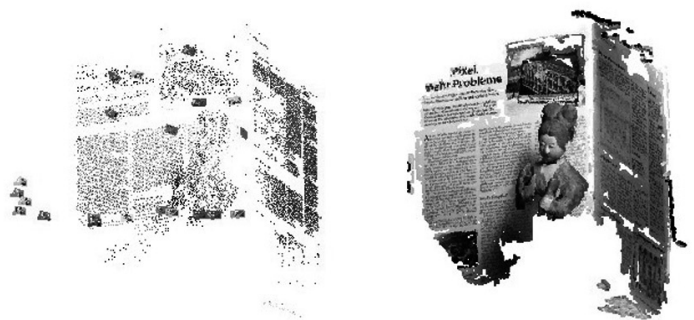

Figure 7: Comparison between sparse and dense point cloud

Patch-based multi view stereo software PVSM and clustering views for multi view stereo CVSM are developments from Yasutaka Furukawa, University of Illionois. Both tools are integrated in the GUI-based structure of VSFM.

\subsection{Online Services}

One of the first online services for structure from motion evaluation was ARC 3D operated by the VISICS group of KU Leuven, Belgium. Following the registration a set of images can be uploaded and the user receives an email upon finishing the job and can upload the textured 3D model designed for further processing with MeshLab.

The idea behind Microsoft's Photosynth is to publish image bundles for viewing and navigating in the photo model. Nonetheless it is possible to download the point clouds, as demonstrated by Pomaska in his CIPA contr Kyoto 2009.

Another free web site service for constructing 3D models from stereo image pairs can be used by visiting www.photo-to3d.com. The software is as well available for Android smartphones. The former Hypr3D is now labeled Cubify Capture.

The most complete and professional system is offered by Autodesk in the 123D suite with 123DCatch. Autodesk offers a complete system to the web community for generating and processing $3 \mathrm{D}$ models. The quality of a high density mesh compared to other sensors is presented here in figure 8. A short video clip is exported to YouTube and can be viewed on: www.youtube.com/watch? $\mathrm{v}=\mathrm{RZWkDqYh} 8 Z 8$. Figure 1 is also a result from 123DCatch.

At this point we can summarize that DCC images evaluated with 123DCatch and Carmine 1.09 recordings with Skanect provide polygonal surfaces for processing the final mesh both meet the requirements. Applying alternatives to a test item gives visual impression of the mesh quality, see figure 8 .

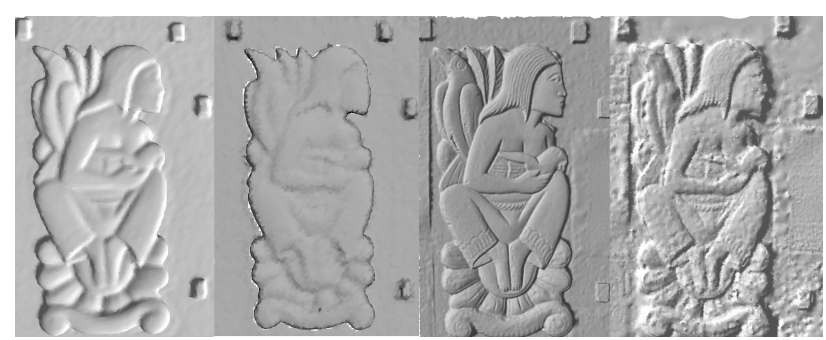

Figure 8: Visual quality check from left to right Carmine 1.09, Kinect, DCC and webcam with 123DCatch

\section{MESH PROCESSING}

The scanning software, mentioned in chapter 2, comes with sophisticated finishing functions to generate a watertight mesh from the raw recording. Despite that, full control to the point cloud requires user interaction and access to the code. The major open source tool for mesh processing is MeshLab. The MeshLab system was developed by ISTI-CNR (Institute of information science and technology - National ResearchCouncil, Italy) in the framework of the EPOCH Network of Excellence. MeshLab provides tools for the processing pipeline from range maps to $3 \mathrm{D}$ polygon models. It includes functionality for registration of different raw range maps, algorithms for surface reconstruction into a single mesh, mesh refinement and editing functions. MeshLab supports a wide range of common $3 \mathrm{D}$ formats, as the polygon file format PLY, the 3D geometry definition of Alias Wavefront OBJ or the surface tessellation language STL.

\subsection{From Point Cloud to Mesh Geometry}

In a first step a point cloud should be prepared for mesh generation. Points of interest must be selected, the number of points may be reduced by resampling. Double vertices should be eliminated and the normal vectors for point sets are required in advance of surface reconstruction. Recommended is the poisson reconstruction with an octree depth around 9 and a solver divide value of around 7. The result should be checked for existing non-manifold faces and holes. Smoothing and simplification provides the final surface geometry. With vertex transfer the colours from the point clouds are transformed to the mesh. Figure 9 shows an item from the lapidarium. At the outlet the point cloud was reduced from 512.000 samples to 113.000 samples. Afterwards the geometry is constructed and 
finally the texture is applied. The process is explained in detail in the tutorial available from the web site related to this paper. Figur 11 demonstrates the conversion of this item from a surface to a solid.
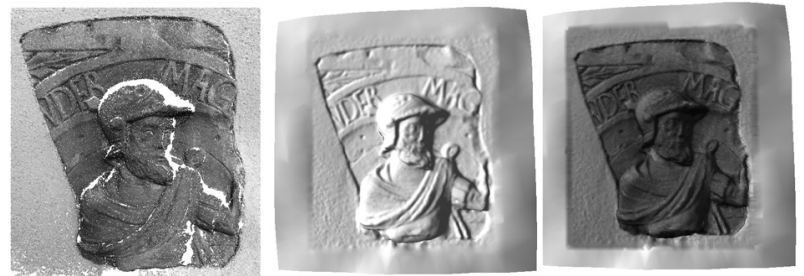

Figure 9: From point cloud to textured mesh

\subsection{Registration and Transformation}

In practice and on site it is difficult to receive a complete scan of a 3D object. Separated scans must be aligned or registered. Meshlab supports the registration with the alignment tool. Single scans are different coloured and merged with the ICP (iterative closest point) algorithm. Since MeshLab works with layers for every mesh, following the alignment the layers should be flatten and a new surface reconstruction can be called. If a textured surface is required, MeshLab enables texturing from vertex colors or the association of raster images. Applying the manipulators tool enables transformation of the mesh to the origin of the surrounding box, rotation and scaling. Finally Meshlab is a perfect tool for viewing and shading meshes. Becoming familiar with MeshLab needs a slightly learning curve. MrP's YouTube channel provides very helpful video clips. A short German tutorial can be downloaded from www.scanner.imagefact.de.
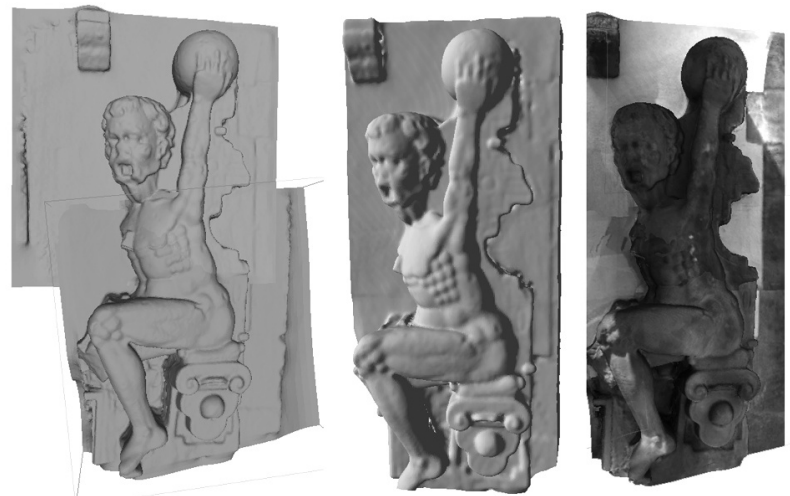

Figure 10: Alignment of two single scans. Left false colours, right textured after vertex colour transformation

\section{FINISHING AND ANALYSING}

\subsection{Repairing and Capping Relief Scans with MeshMixer}

As one can detect in figure 1 some of the stone work show only the front surface due to wall integration. To receive a closed or watertight model we need some further processing. The boundary for the relief shape must be found, extruded and closed by capping. MeshMixer (www.meshmixer.com) from Autodesk is designed for modelling 3D objects and finishing of scanned items. The inspection tool detects holes, non-manifold areas and disconnected components. After repairing those artefacts the boundary of the mesh is detected and displayed. A selected boundary can be smoothed and extruded within the modify menu by moving a slider in the toolbox or typing the distance. Finally the extrusion can be scaled and filled with the erase and fill function before exporting as an STL-File (standard tessellation language) for analysing or printing. Figure 11 (a screenshot of MeshMixer) displays a scan prepared with Meshlab. The surface is shown from behind with an extrusion of the boundary but still not closed.

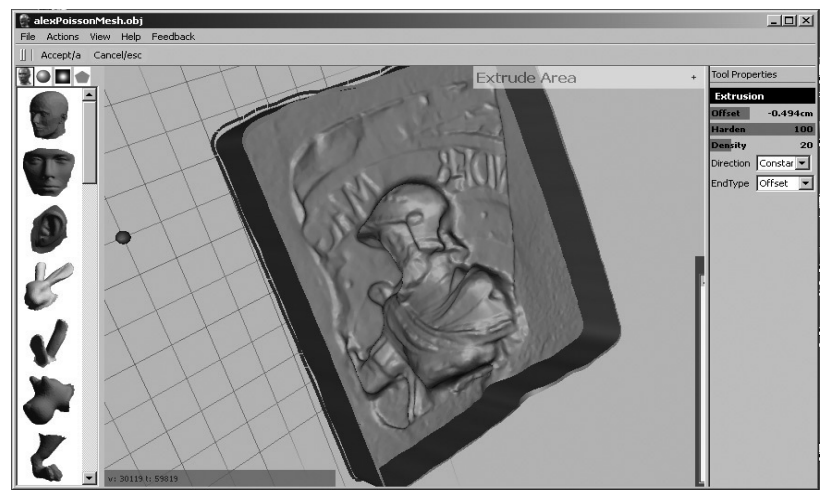

Figure 11: Finding a smooth boundary and capping a mesh with MeshMixer

\subsection{Analysing a Mesh with Preprocessors for 3D Printing}

Preprocessors for 3D printing are responsible for analysing the mesh, editing, cutting, scaling and positioning objects on the virtual printer plate and sending the G-Code to the printer interface. Netfabb Studio Professional (www.netfabb.com) provides powerful features bringing meshes to printer output. Netfabb Studio Basic is not only a trial software, it is the basic version of Netfabb Studio made available free of charge for the convenience of students, teachers, private individuals and professionals who do not require the advanced features of Netfabb Studio Professional. Despite we do not focus on 3D output to hold the stone work in our hand, the manufacturing software provides valuable functions for inspecting the gems necessary for the restoration process as there are dimensioning, intersection or slicing. Figure 12 illustrates the slicing process. Lines are presented in the $\mathrm{z}$ and $\mathrm{y}$ direction while a full cut is shown in $\mathrm{x}$ direction. The user can adjust the distance of slices, view a slicing animation and can output the contours to a SVG file. Furtermore the measurement function may be very helpful for dimensioning distortions in the model.

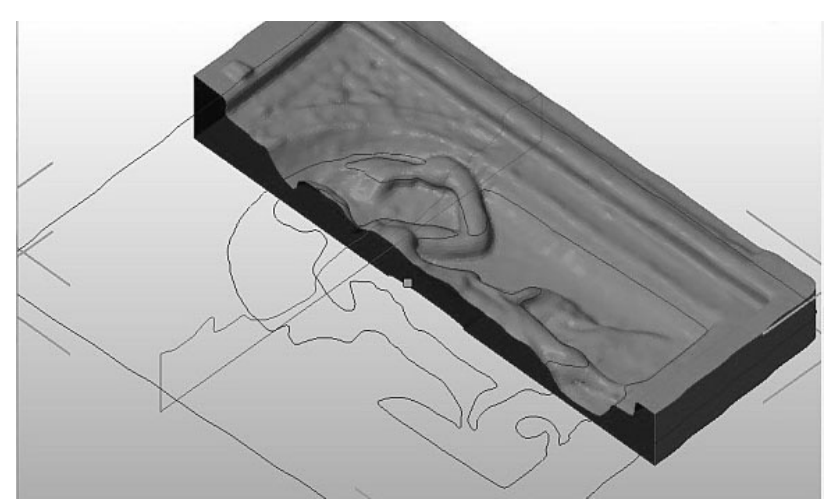

Figure 12: Netfabb slicing tool 


\section{3}

Little Helpers

Due to today's hype in 3D printing several free software tools for preprocessing 3D models are published. To a certain extend we can benefit from those tools in our documentation project. For example there is SLIC3R, ReplicatorG or MakerWare which are operating as drivers for 3D printers. G-Code is the language for driving machines and generate paths for the tool movement by slicing the virtual object. The output files assist digital interfacing for drawing in SVG for example. Makerware, the software for driving the Replicator 3D printer, enables easily handling of rotation, positioning and scaling of meshes and external storage of the executed transformations.

State of the art for presenting interactive 3D models on web sites is WebGL. WebGL is native integrated in modern browsers. Making things easy, one can upload and share his models with the community via Sketchfab. A link to Sketchfab on own sites into Iframes make use of the Sketchfab hosting.. Examples are given on the web site related to this paper.

Other software projects as Cloud Compare and $\mathrm{CC}$ viewer or Graticulate 3D a free network adjustment project complete the list of usable tools. But the latter are more sophisticated. And finally Blender the open source project for rendering and animation should be taken in consideration for graphical presentations in computer environments. The great Blender project is much more as a little helper. In our project Blender is used for high end rendering and as a powerful presentation tool.

\section{SUMMARY AND CONCLUSION}

$3 \mathrm{D}$ object reconstruction today is a technology given to non-experts applying low-cost scanning equipment like digital cameras and depth cameras connected to a notebook computer. Recording software is available as freeware or licensed for low budgets. Open Source tools like MeshLab and Blender and to a certain extend the functionality of $3 \mathrm{D}$ printing software enables finishing a scanned surface, analysing and presentation. Limitations of the here described configuration depend on the object's volume and the midrange level accuracy.

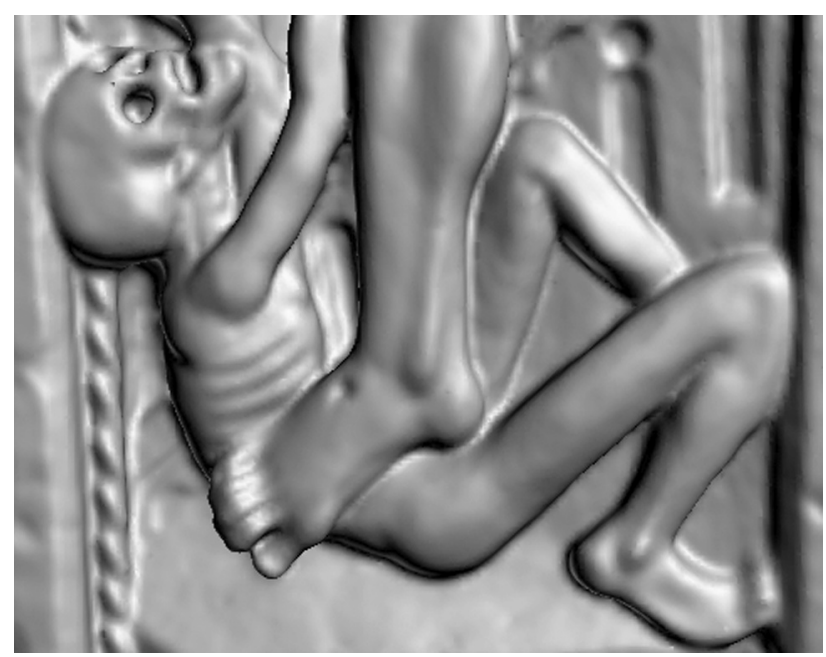

Figure 13: Cutout of a perron with resurrection scene after an original from Albrecht Dürer
A laptop computer equipped with a 7core NVIDIA graphics card and 8GByte of Ram is recommended. In case of textured items the digital camera provides perfect results. If the surface is not textured Carmine 1.09 is a suitable. In case of specular surfaces some preparation with matt finishing is required. Talking about accuracy often overestimates the client's real requirement. In case of high demands the appropriate scan devices should be applied.

By handing over, in addition to the surface models, recording rules and software tutorials the process of $3 \mathrm{D}$ recording may be integrated into the museum's infrastructure and assist the documentation and restoration process. The web site www.scanner.imagefact.de is related to this contribution.

\section{References from Books:}

N.N., 2013. Ultimate Guide to 3D Printing. Make, O'Reilly

Borenstein, G., 2012. Making Things See. O'Reilly, Sebastopol, CA

\section{References from Other Literature:}

Kazhdan, M., et.al., 2006. Poisson Surface Reconstruction. Eurographics Symposium of Geomtry Processing, 2006

Kofler, M., 2011. Inbetriebnahme und Untersuchung des Kinect Sensors. Masterprojekt FH Oberösterreich

Newcomb, R., et.al., 2011. KinectFusion: Real-Time Dense Surface Mapping and Tracking. Microsoft Research

Pomaska, G., 2013. 3D-Rekonstruktion unstrukturierter Objekte. 12. Internationales 3D-Forum Lindau

Pomaska, G., 2009. Utilization of Photosynth Point Clouds for 3D Object Reconstruction. CIPA International Symposium Kyoto, Japan

Reinhardt, M., et.al., 2011. 3D-Scan 2.0. Technische Universität Bergakademie Freiberg

Schares, J.M, et.al., 2012. Geometrische Untersuchungen zur Tiefengenauigkeit des Kinect-Sensorsystems. DGPFJahrestagung

References from web sites:

Hardware and Device Drivers: http://www.primesense.org

Scanning Software:

http://www.cs.washington.edu/homes/ccwu/vsfm http://reconstructme.net

3D Modelling:

http://meshlab.sourgeforge.net

http://www.meshmixer.com

http://pixologic.com/sculptris/

http://www.Blender.org

3D Printing:

http://www.netfabb.com

http://replicat.org

http://www.sketchfab.com

http://i.materialise.com

http://www.3ders.org 